\title{
Tratamento de assimetria facial causada por hiperplasia condilar
}

\author{
Treatment of facial asymmetry caused by condilar hyperplasia
}

\author{
MARIANA FERREIRA Silva Ventura ${ }^{1}$ \\ EUGÊNIO BRAZ RODRIGUES ABRANTES ${ }^{2}$ \\ RODRIGO FIGUEIREDO DE BRITO RESENDE ${ }^{3}$ \\ MARCELO José PINHEIRO GUEDES DE UZEDA ${ }^{4}$ \\ RAFAEL SEABRA LOURO
}

\section{RESUMO}

Introdução: A hiperplasia condilar é uma condição rara do desenvolvimento ósseo causada pela hiperatividade do côndilo mandibular que resulta em seu crescimento excessivo e autolimitado. Caracterizase pelo alongamento progressivo do processo condilar levando à assimetria facial, alongamento do terço inferior da face, má oclusão e ocasionalmente disfunção temporomandibular. Diante das formas de tratamento, o preparo ortodôntico associado à cirurgia ortognática e a condilectomia apresentam resultados satisfatórios.

Objetivo: O objetivo deste trabalho é apresentar o caso clínico de um paciente do sexo masculino, 28 anos de idade, portador de hiperplasia condilar unilateral com assimetria facial severa, protrusão mandibular e desvio do mento.

Relato de caso: O tratamento foi realizado no Hospital Federal dos Servidores do Estado/RJ,por meio de cirurgia ortognaticamaxilomandibular com mentoplastia associada à condilectomia alta, planejada previamente utilizando modelos estereolitográficos. A correção da deformidade esquelética, por intermédio da cirurgia ortognática, efetuou expressiva melhora estético-funcional do paciente.

Conclusão: O mesmo se encontra em controle pós-operatório de seis anos, sem recidiva e com um padrão de oclusão satisfatório.

Palavras-chave:Côndilo Mandibular Hiperplasia.Cirurgia.

\begin{abstract}
Introduction: Condylar hyperplasia is a rare condition of bone development caused by hyperactivity of the mandibular condyle resulting in its excessive and self-limited growth. It is characterized by progressive elongation of the condylar process leading to features such as facial asymmetry, elongation of the lower third of the face, malocclusion and occasionally temporomandibular dysfunction. In view of the forms of treatment, the orthodontic preparation associated with orthognathic surgery and the condilectomy present satisfactory results.

Objective: The objective of this study is to present the case of a 28 year old male patient with unilateral condylar hyperplasia with severe facial asymmetry, mandibular protrusion and deformity of the chin.

Case report:The treatment was performed at the Federal Hospital of the State Servicers/RJ, through orthognathicmaxilomandibular surgery with mentoplasty associated with high condilectomy, previously planned using stereolithographic models. Conclusion: The correction of the skeletal deformity, through orthognathic surgery, effected an expressive aesthetic-functional improvement of the patient. The same is in postoperative control of six years, without relapse and with a satisfactory occlusion pattern.
\end{abstract}

Keywords: Mandibular condyle. Hyperplasia. Surgery.

1 Graduada em Odontologia pela Universidade Federal Fluminense, Niterói / RJ, Brasil.

2 Especialista em Estomatologia pela Universidade Federal do Rio de Janeiro, Rio de Janeiro / RJ, Brasil e Residente do Serviço de Cirurgia e Traumatologia Bucomaxilofacial da Universidade Federal Fluminense / Hospital Federal dos Servidores do Estado, Rio de Janeiro / RJ, Brasil.

3 Mestre e Doutor em Odontologia pela Universidade Federal Fluminense, Niterói / RJ, Brasil e Professor das disciplinas de Anestesiologia e Cirurgia Oral Menor da Universidade Federal Fluminense, Niterói / RJ, Brasil.

4 Mestre e Doutor em Odontologia pela Universidade Federal Fluminense, Niterói / RJ, Brasil e Professor das disciplinas Cirurgia Bucal I, II e III da Universidade Iguaçu, Rio de Janeiro / RJ, Brasil.

5 Doutor em Cirurgia e Traumatologia Bucomaxilofacial pela Universidade Estadual de Campinas, Campinas / SP, Brasil e Professor/ Coordenador das disciplinas de Anestesiologia e Cirurgia Oral Menor da Universidade Federal Fluminense, Niterói / RJ, Brasil. 


\section{INTRODUçÃo}

A hiperplasia condilar $(\mathrm{HC})$ é uma anomalia de crescimento rara, caracterizada pelo desenvolvimento excessivo unilateral do processo condilar, ramo e corpo da mandíbula que, em variados graus, causa assimetria dentofacial e maloclusão dentária., ${ }^{1,2,3}$ Geralmente,essa se manifesta em pacientes jovens como resultado de uma hiperatividade do centro de crescimento condilar ou após o final da puberdade quando esse crescimento persiste até a fase adulta, sendo encontrada com mais frequência entre indivíduos dos 11 aos 30 anos de idade..$^{3,4}$

Estudos mostram relação entre a patologia e traumatismos seguidos de excessiva proliferação celular de reparação, resposta à infecção local, influências hormonais, artrose, distúrbios vasculares locais, possíveis condições genéticas e fatores de crescimento. ${ }^{2,3}$ A HC se apresenta sob duas diferentes formas clínicas: o alongamento hemimandibular ou tipo 1,mais comum em adolescentes e adultos jovens, possui vetor de crescimento horizontal com desvio do mento para o lado não afetado e; a hiperplasia hemimandibular ou tipo 2, acomete pacientes mais velhos exibindovetor de crescimento vertical e é caracterizada por mordida aberta ou crescimento compensatório da maxila do lado envolvido causando inclinação do plano oclusal e pouco desvio do mento; há ainda um terceiro tipo que seria a forma mista entre os dois primeiros. ${ }^{1}$

A maioria dos estudos aponta que a $\mathrm{HC}$ possui prevalências similar entre os gêneros em variados grupos étnicos, ${ }^{2,4,5}$ porém há descrições que apontam uma frequência maior em mulheres entre o número de pacientes, dado que é associado às evidências de que a hiperplasia pode ser estimulada por hormônios femininos. ${ }^{7,9,5}$ A severidade dos casos não se encontra ligada ao gênero nem à idade, apesar dos estudos indicaremque as característicashistomorfológicas mudam com o envelhecimento do paciente. ${ }^{3}$

O diagnóstico de hiperplasia condilar pode ser estabelecido por meio dos achados clínicos e de exames de imagem. O estudo inicial através das radiografias panorâmica, lateral e póstero-anterior, demonstra a discrepância no tamanho dos côndilos e o alongamento do corpo condílico. ${ }^{5}$ A cintilografia óssea é uma ferramenta importante no diagnóstico da $\mathrm{HC}$ e no planejamento para o tratamento do paciente acometido. O método identifica se há persistência do crescimento condilar por intermédio da captação dos isótopos radioativos que, uma vez injetados no paciente, se fixarão nos cristais de hidroxiapatita e cálcio demarcando locais onde há intensa atividade osteoblástica. A comparação entre o crescimento ósseo dos côndilos direito e esquerdo concluirá se há hiperplasia condilar ativa. Nos indivíduos normais, a diferença de captação entre os côndilos não passa de $10 \%$. 2,6 A tomografia computadorizada possibilita o estudo tridimensional das relações anatômicas de maxila e mandíbula do paciente.

A correção cirúrgica para a assimetria facial causada pela hiperplasia condilar é um desafio dentro da especialidade e várias estratégias para o seu tratamento foram propostas até hoje. Desde a condilectomia alta até a abordagem cirúrgica bimaxilar e correções de tecido mole em alguns casos e associações dessas abordagens. ${ }^{7,8}$ Nos casos em que a HC está ativa, o planejamento cirúrgico deve abarcar a retirada do centro de crescimento ósseo principal da mandíbula, por meio da condilectomia alta. Posteriormente a esse ou em um único tempo cirúrgico realiza-se a cirurgia ortognática, normalmente bimaxilar para corrigir o padrão facial ainda inadequado após o primeiro ato cirúrgico. ${ }^{9}$

O objetivo deste trabalho é apresentar ocaso clínico de um paciente portador de hiperplasia condilar ativa tratado por meiode cirurgia ortognática associada à condilectomia.

\section{ReLATO de CASO}

Paciente do sexo masculino, 28 anos de idade, compareceu ao Serviço de Cirurgia Oral e Maxilofacial do Hospital Federal dos 
Servidores do Estado/RJ, queixando-se de "sorriso torto e mordida errada". Durante a anamnese,se queixava de dor na região da articulação temporomandibular direita aos movimentos de abertura da boca, sem história de trauma ou infecção. Ao exame físico extraoral, observou-se assimetria facial severa, protrusão mandibular associada a desvio do mento para o lado esquerdo, aumento e alongamento da altura vertical dos terços médio e inferior de face do lado direito (Figura 1 -A). Em uma vista inferior do mento, percebeu-se curvatura do corpo mandibular com expressivo deslocamento do mento para - lado esquerdo e da basilar da mandíbula (Figura 1 - C). Ao exame intraoral,verificouse assimetria do plano oclusal (Figura $1-B$ ), mordida de topo associada à mordida aberta no lado direito e desvio da linha média mandibular de $3 \mathrm{~mm}$ para o lado esquerdo, relação de oclusão de molar do tipo I de Angle no lado esquerdo e aos movimentos de abertura e fechamento bucal, latero-desvio para a direita.

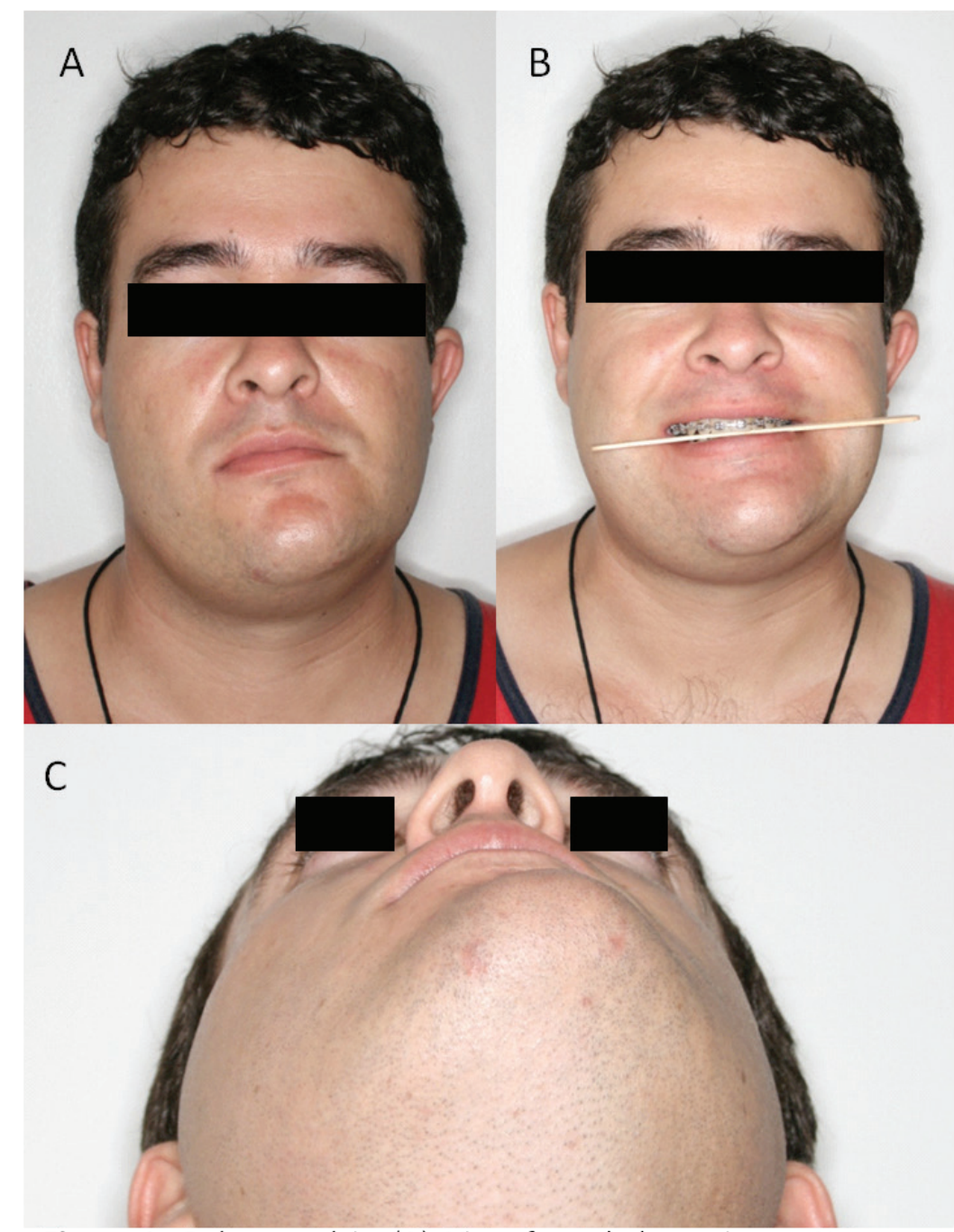

Figura 1: Pré-operatório (A) Vista frontal do paciente. Nota-se o alongamento unilateral do terço inferior direito de face gerando a assimetria facial e o desvio do mento; (B) Espéculo de madeira entreposto entre os dentes demonstrando a angulação do plano oclusal; (C) Vista inferior do mento evidenciando expressivo desvio lateral da linha média. 
Os exames prévios de radiografia panorâmica, póstero-anterior, cefalométrica e tomografia computadorizada revelaram aumento significativo do processo condilar com alongamento e espessamento do colo do côndilo do lado direito e aparência normal do lado esquerdo (Figura 2). A morfologia da cabeça do côndilo afetado era uniforme, apesar de aumentada. O ramo e corpo mandibular apresentavam-se alongados em direção inferior e o longo eixo da superfície lateral do ramo direito era alterado em relação ao do lado não afetado em uma vista frontal. O ângulo mandibular do lado afetado se encontrava inferior e posterior comparado ao contralateral. A espessura do corpo mandibular era maior do lado não afetado em comparação ao afetado. Durante a avaliação da cintilografia óssea foi possível evidenciar um aumento da atividade metabólica no côndilo direito, sugerindo que a doença ainda se encontrava em atividade.
Para o tratamento da assimetria causada pela hiperplasia condilar, optou-se pelo acompanhamento ortodôntico prévio para o preparo ortocirúrgico. A Tomografia Computadorizada FanBeam foi realizada para caracterizar o quadro clínico. Os cortes tomográficos mostraram diferenças entre as cabeças condilares e se confirmou o alongamentodo pescoço do côndilo direito. A tomografia computadorizada também foi utilizada para o planejamento virtual do procedimento cirúrgico, por meio dos dados tomográficos foram confeccionados modelos estereolitográficos para a simulação cirúrgica prévia com o objetivo de reproduzir fielmente todos os detalhes anatômicos, os movimentos e os cortes cirúrgicos a serem realizados. As características clínicas e radiográficas concordaram parao diagnóstico de côndilo hiperplásico unilateral do lado direito.

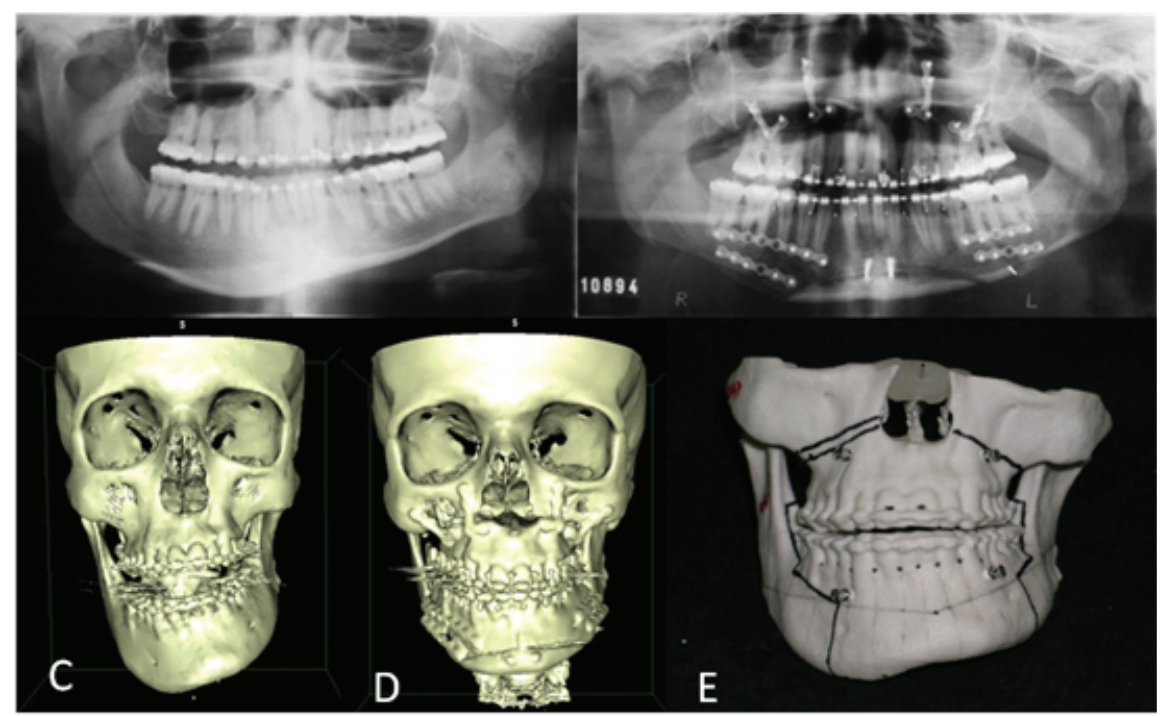

Figura 2: (A) Radiografia panorâmica pré-operatória, nota-se a discrepância entre o tamanho dos côndilos, ramo e corpo mandibulares do lado direito; (B) Radiografia panorâmica de controle pós-operatório mostrando a boa simetria mandibular; (C) Tomografia computadorizada pré-operatória (reconstrução 3D) em vista anterior evidenciando a assimetria e alongamento das estruturas anatômicas do paciente; (D) Tomografia computadorizada (3D) pós-operatória demonstrando a melhora da relação anatômica entre maxilares; (E) Biomodelo prototipado obtido dos dados da tomografia computadorizada e marcações das osteotomias planejadas para ocaso. 
O plano de tratamento incluiu duas fases cirúrgicas para a remoção da doença condilar e devolução da função. A primeira fase inicial tratou-se da condilectomia alta seguida pela segunda fase do tratamento ortocirúrgico por meio de cirurgia ortognática combinada. O procedimento cirúrgico foi realizado sob anestesia geral, com intubação nasotraqueal, onde se realizou a ressecção condilar unilateral no lado direito para remover o local de crescimento. Em seguida, foi feita cirurgia ortognática com osteotomia Le Fort I maxilar e osteotomia sagital bilateral de mandíbula que respeitaram a relação maxilomandibular planejada, com o auxílio de acessório interoclusal de resina acrílica termopolimerizável obtido por meio dos biomodelos manipulados em articulador semiajustável. Para a melhora da correção da protrusão mandibular foi feita mentoplastia associada ao recuo.

A cirurgia foi realizada em centro cirúrgico, sob anestesia geral, utilizando intubação nasotraqueal com tubo aramado fixado à base alar com fio de sutura número 2.0. Após a antissepsia intra e extraoral do paciente e posicionamento dos campos cirúrgicos estéreis, realizou-se o tamponamento orofaríngeo. Foi feita infiltração anestésica de lidocaína $2 \%$ com vasoconstritor 1:1000000 em fundo de sulco vestibular e extraoralmente em subcutâneo de região pré-auricular, sítios de incisão de acesso. Incisão com lâmina de bisturi número 15 executada em região préauricular direita e dissecção por planos com atenção para o ramo temporal do nervo facial mantendo cuidado com o acesso às estruturas articulares do lado direito. Performou-se então a osteotomia com instrumentos rotatórios na região superior da cabeça condílica do lado direito e, ao final,sutura por planos.

Realizou-se a troca de luva entre os cirurgiões para mudançanos acessos cirúrgicos intra e extraorais. Acesso vestibular mandibular posterior para osteotomias sagitais bilaterais em mandíbula com instrumentos rotatórios e cinzel reto de $10 \mathrm{~mm}$ de largura.Posicionamento de goteiraee splint intermaxilar confeccionado previamente com resina autopolimerizável para guia de oclusão intermediário retido com bloqueio maxilomandibular temporário com fio de aço. Foi feita então a fixação dos segmentos mandibulares com placas e parafusos do sistema $2.0 \mathrm{~mm}$ (DePuySynthes) seguida da retirada do bloqueio maxilomandibular e remoção da goteira intermediária.

O acesso vestibular maxilar com dissecção mucoperiosteal delicada para a região de septo e região nasal para realização das osteotomias, horizontais em maxila (Le Fort I) com instrumentos rotatórios e cinzel curvo e para osteotomia do processo pterigoide da maxila. Com um auxílio de um instrumental delicado ocorre rebaixamento do bloco da maxila solto de sua posição de origem. Posiciona-se a goteira de palato confeccionada previamente com resina autopolimerizável para realização de "Down-fracture" completo da maxila por meio de fórceps de Rowe e reposição inferior da maxila seguida da retirada da goteira de palato.

Instalação da goteira final feita previamente com resina autopolimerizável fixada com fios de aço no aparelho ortodôntico superior. É feita a confirmação da dimensão vertical facial do paciente por meio de marcação em glabela realizada previamente. Após o bloqueio maxilomandibular com fios de aço é feita a fixação da maxila com placas e parafusos do sistema $2.0 \mathrm{~mm}$ (DePuySynthes ${ }^{\circledR}$ ) seguida de remoção do bloqueio maxilomandibular e da goteira final. Confirma-se a oclusão final desejada e, ao final, realiza-se a síntese dos tecidos com categute cromado $3.0 \mathrm{em}$ mandíbula e categute simples 3.0 em maxila.

Como resultado do procedimento, obtevese expressiva melhora estética com a correção da assimetria e ganho de estabilidade da oclusão (Figura 3). O paciente se encontra em acompanhamento há 6 anos sem recidiva e não relata queixas de dor ou disfunção temporomandibular. 


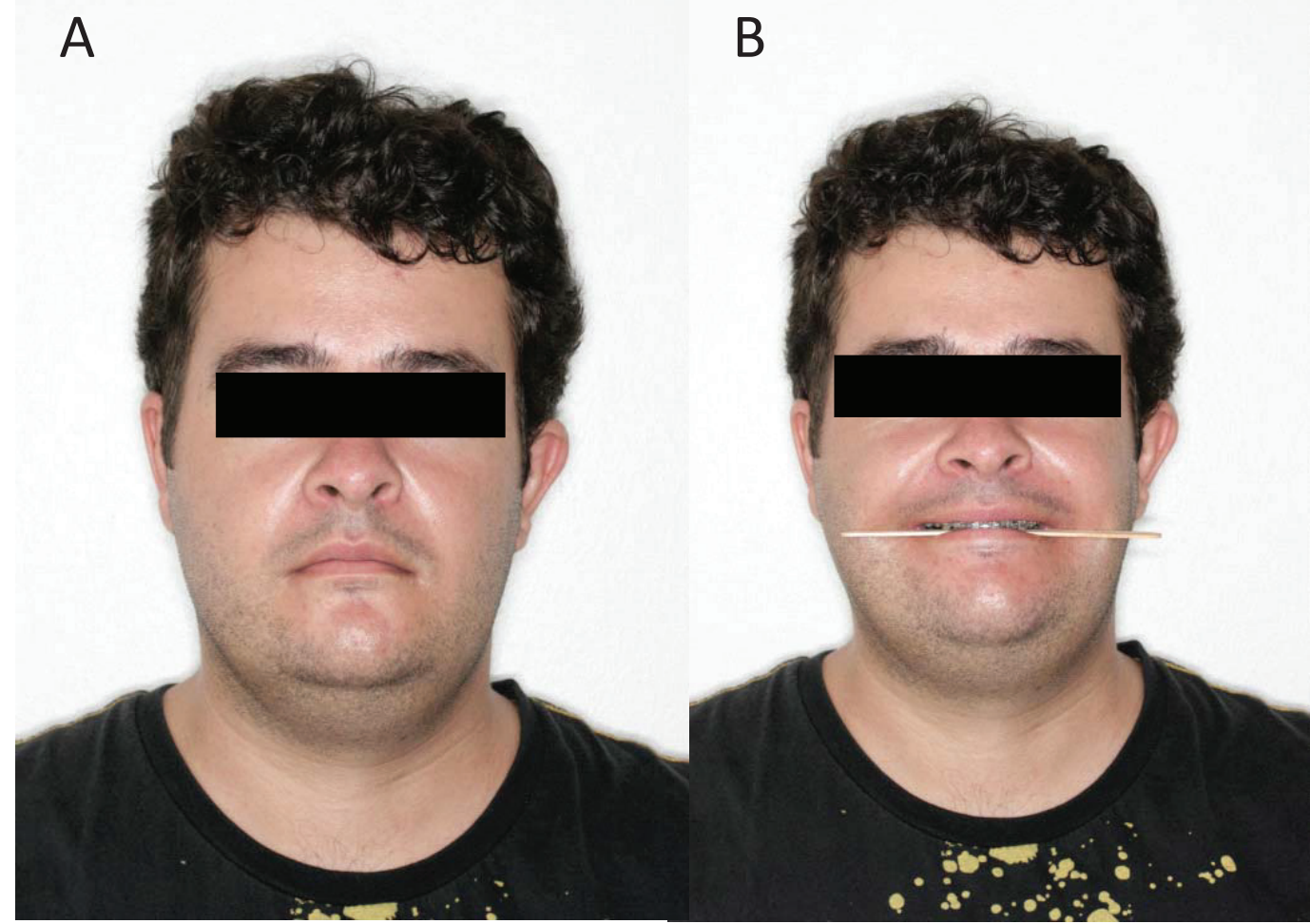

Figura 3: (A) Controle pós-operatório, vista frontal, nota-se a resolução da assimetria causada pela Hiperplasia Condilar; (B) Espéculo de

\section{DIscussão}

A HC é reconhecidamente uma patologia cujo plano de tratamento é complexo e desafiador. Aliteratura diverge na determinação de sua terapêutica, pois há dificuldade em relacionar a morfologia patológica, o estágio de avanço em que se encontra e o prognóstico. Para o manejo adequado, é imprescindível o diagnóstico preciso de cada caso. Não há consenso a respeito da preferência por acometimento de algum dos lados, e há divergência entre as pesquisas sobre qual gênero é mais afetado. ${ }^{2} \mathrm{E}$ apesar de existir a relação entre o lado direito ser mais afetado em mulheres e o esquerdo em homens, ${ }^{5}$ no presente caso trata-se de um homem acometido do lado direito.
A HC possui diagnóstico diferencial com outras patologias, principalmente com - osteocondroma que se assemelha na assimetria progressiva e mudanças da oclusão do paciente, sendo até mesmo considerado um subtipo da hiperplasia condilar na literatura, ${ }^{9}$ porém a maioria dos autores os considera patologias distintas e, na nossa experiência, não é a classificação mais desejável por se tratarem histologicamente de patologias diferentes com comportamentos distintos.

A investigação através de imagens é fundamental, pois o tratamento se apoia sobre o exame minucioso dos detalhes anatômicos de cada caso. Para auxílio do plano operatório, pode-se lançar mão da prototipagem dos maxilares. ${ }^{9}$ Além disso, a investigação da presença de atividade de crescimento influencia diretamente na determinação do 
plano de tratamento e, para tal, usualmente complementa-se o planejamento com a cintilografia ou com tomografia por emissão de pósitrons (SPECT) que quantificará a absorção do isótopo radioativo pelos osteoblastos em atividade dos côndilos. Nos indivíduos normais, a diferença de um côndilo para outro está entre $3 \%$ e $5 \%$ e se define a doença como ativa quando esse valor ultrapassa $10 \%,{ }^{2,6} \mathrm{~A}$ investigação de atividade da patologia é fundamental para decisão do tratamento, a partir da confirmação dela optouse pela realização da condilectomia no caso apresentado, a fim de remover o centro de crescimento e reprimir a recidiva da assimetria facial após o tratamento.

Diversas formas de tratamento foram propostas para a Hiperplasia Condilar a depender de como se apresenta cada caso. O tratamento ortocirúrgico visa restabelecer a boa conformidade facial e o equilíbrio oclusal de maneira previsível e estável. A correção ortodôntica é ideal para o preparo e a finalização dos casos, alinhando os dentes e descompensando as arcadas, proporcionando um planejamento preditivo, ou seja, uma abordagem multidisciplinar beneficiará o paciente quando a intervenção está indicada. No caso exibido, o paciente apresentou oclusão final ótima devido ao preparo ortodôntico prévio. ${ }^{9}$

Quandoé detectadaa presença de atividade de crescimento em um dos côndilos existe a opção de adiar a cirurgia até a autolimitação da patologia para, a partir de então, planejar as devidas correções, porém esta é uma alternativa que, apesar de aplicável para casos menos severos, deixa o paciente a sofrer das consequências da doença por um longo período sem que haja uma maneira de prever quando a hiperatividade cessará, por esse motivo não optamos por essa possibilidade..$^{9,10,11}$ Assim, como alguns autores preconizam, preferimos intervir cirurgicamente tão logo quanto possível a fim de conter o crescimento exacerbado e permitir um desenvolvimento facial mais adequado..$^{7,8,9,11}$ Apenas cirurgia ortognática não é a opção de tratamento aceitável para esses pacientes, pois a continuidade do crescimento torna os resultados instáveis. ${ }^{7}$

O tratamento indispensável para os casos ativos é a condilectomia total ou alta, retirando de 3 a $5 \mathrm{~mm}$ da parte superior estendendo a osteotomia para medial e lateral do côndilo. ${ }^{7,9}$ Há debates a respeito da cirurgia para a realização da condilectomia total ou alta simultaneamente com a ortognática ou num segundo tempo cirúrgico. Alguns autores decidem pela ortognática apenas após a certeza de que o centro de crescimento foi inteiramente removido, obtendo assim um resultado mais estável em longo prazo, porém essa opção submete o paciente a uma segunda intervenção, o que pode ser uma dificuldade de aceitação. ${ }^{10} \mathrm{~A}$ opção concomitante das duas técnicas, como no caso apresentado, ao reduzir o tamanho do côndilo proporciona uma rotação cranial do fragmento sendo favorável para a correção de parte da assimetria, além de remover o foco da patologia prevenindo a recidiva se mostrando estável em longo prazo.

Os pacientes comprovadamente sem atividade hiperplásica podem ser submetidos apenas a correção cirúrgica da assimétrica com a cirurgia ortognática. Após a compensação da oclusão, alguns refinamentos podem ser necessários, como a mentoplastia e a redução da borda inferior do corpo mandibular, para boa adequação dos tecidos moles e recuperação estética satisfatória. ${ }^{7,9,10}$ Os casos em que há disfunção da articulação temporomandibular com deslocamento do disco requerem o reposicionamento e ancoramento do mesmo ao ramo condilar. ${ }^{10}$

\section{CONSIDERAÇÕES FINAIS}

O tratamento para a assimetria facial resultante da hiperplasia condilar é um dos 
grandes desafios do cirurgião bucomaxilofacial. O auxílio dos exames de cintilografia óssea e o planejamento por meio de biomodelos gerados pelos dados da tomografia computadorizada possibilitam um diagnóstico mais preciso e um melhor delineamento adequado a cada caso. A associação da condilectomia com a cirurgia ortognática demonstrou ser estável e previsível por devolver a função e diminuir a alteração estética do paciente.

\section{REFERÊNCIAS}

1. Obwegeser HL, Makek MS. Hemimandibular hyperplasia - Hemimandibular elongation. J Maxillofac Surg. 1986, Aug;14(4):183-208.

ht t p s://www.ncbi.n Im.nih.gov/m/ pubmed/3461097/

2. Gray RJ, Sloan P., Quayle AA, et al. Histopathological and scintigraphic features of condylar hyperplasia. Int J Oral Maxillofac Surg. 1990, Apr;19(2):65-71.

https://www.ncbi.nlm.nih.gov/m/ pubmed/2111360/

3. Slootweg PJ, Muller H: Condylar hyperplasia: A clinico-pathological analysis of 22 cases. J Maxillofac Surg. 1986, Aug;14(4):209-14.

https://www.ncbi.nlm.nih.gov/m/ pubmed/3461098/

4. Eslami B., Behnia H., Javadi $H_{\text {., }}$ et al. Histopathologic comparison of normal and hyperplastic condyles. Oral Surg Oral Med Oral Pathol Oral RadiolEndod. 2003, Dec;96(6):711-7. ht t p s://www.ncbi.nIm.nih.gov/m/ pubmed/14676762/

5. Nitzan DW, Katsnelson A., Bermanis I., et al: The clinical characteristics of condylar hyperplasia: Experience with 61 patients. J Oral Maxillofac Surg. 2008, Feb;66(2):312-8. doi: 10.1016/j. joms.2007.08.046.

ht tps://www.ncbi.nIm.nih.gov/m/ pubmed/18201615/
6. Saridin CP, Raijmakers P., Becking AG. Quantitative analysis of planar bones cintigraphy in patients with unilateral condylar hyperplasia. Oral Surg Oral Med Oral Pathol Oral Radiol Endod. 2007, Aug;104(2):259-63. Epub 2006, Dec 4.

https://www.ncbi.nIm.nih.gov/m/ pubmed/17142067/

7. Villanueva-Alcojol L., Monje F., González-García R. Hyperplasia of the Mandibular Condyle: Clinical, Histopathologic, and Treatment Considerations in a Series of 36 Patients. J Oral Maxillofac Surg. 2011, Feb;69(2):447-55. doi: 10.1016/j.joms.2010.04.025. Epub 2010, Sep 9.

https://www.ncbi.nIm.nih.gov/m/ pubmed/20828911/

8. Ferguson, JW. Definitive surgical correction of the deformity resulting from hemimandibular hyperplasia. J Craniomaxillofac Surg. 2005, Jun;33(3):150-7. Epub 2005, Apr 22.

https://www.ncbi.nIm.nih.gov/m/ pubmed/15878514/

9. Wolford LM, Movahed R., Perez DE. A classification system for conditions causing condylar hyperplasia. J Oral Maxillofac Surg. 2014, Mar;72(3):567-95. doi: 10.1016/j. joms.2013.09.002. Epub 2014, Jan 1.

https://www.ncbi.nIm.nih.gov/m/ pubmed/24388179/

10. Olate S., De Moraes M. Deformidad facial asimétrica. Papel de la hiperplasia condilar. Int J Odontostomat. 2012; 6(3): 337-347.

http://dx.doi.org/10.4067/S0718381X2012000300017

11. Martinez, $\mathrm{H}$. et al. Management criteria for condilar hiperplasia: a personalized treatment for each type of condition. International Journal of Oral and Maxillofacial Surgery, v.48, 172.

https://doi.org/10.1016/j.ijom.2019.03.534

Submetido em:14-6-2019

Aceito em: 02-03-2020 